\title{
Preliminary study on the In-Vitro Susceptibility of Mycobacterium tuberculosis Isolates to Virgin Coconut Oil
}

\author{
Godofreda V. Dalmacion ${ }^{1}$, Adelwisa R.Ortega ${ }^{2}$, Imelda G. Pena ${ }^{3}$, and Concepcion F. \\ Ang $^{4}$
}

${ }^{1}$ University of the Philippines-Manila, College of Medicine, 547 Pedro Gil, Ermita 1000, Manila, Philippines; ${ }^{2}$ University of the Philippines-Manila, College of Public Health, 547 Pedro Gil, Ermita 1000, Manila, Philippines; ${ }^{3}$ University of the Philippines, College of Pharmacy, Taft Avenue, Ermita 1000, Manila, Philippines; ${ }^{4}$ University of the Philippines-Philippine General Hospital, Taft Avenue,Ermita 1000, Manila, Philippines

*Corresponding author: Godofreda V. Dalmacion, MD, Professor, Department of Pharmacology and Toxicology, College of Medicine, University of the Philippines, Ermita 1000, Manila, Philippines

Submission date: July 9, 2012, Acceptance date: August 28, 2012; Publication date: August 30, 2012

\section{ABSTRACT:}

Background: Tuberculosis remains a major public health threat. Studies have shown that medium chain fatty acids (MCFA), such as, those found in Virgin Coconut Oil (VCO), possess activity against a wide range of microorganisms including Mycobacterium tuberculosis. The goal of the study was to determine the in-vitro susceptibility of M. tuberculosis (TB) isolates to two commercial brands of VCOs each containing different amounts of lauric acid and produced by two different extraction processes. Two brands of VCO were tested for their anti-TB actions compared to pure lauric acid and a negative control. Two replicate runs were done. VCO samples were inoculated with $0.1 \mathrm{~mL}$ of the H37RV strain of M. tuberculosis and incubated at $37^{0} \mathrm{C}$ with shaking for 5 days. After incubation, the mixtures were diluted 10 -fold up to $10^{-4}$ concentration to obtain countable colonies using Middlebrook 7H10 agar plates and LowensteinJensen (L-J) slants. Colony growths of M. tuberculosis were examined in each dilution every week for three to eight weeks.

Results: Both brands of VCO at increasing dilution from 1:10 to 1:10000 lowered the colony counts of $M$. tuberculosis by $46 \%$ to $100 \%$ on the Middlebrook 7H10 agar. Similar results were noted on L-J slants with colony counts decreasing by $25 \%$ to $96 \%$. Lauric acid solution showed complete inhibition of bacterial growth in both the agar plates and LJ slants.

Conclusion: Different VCO preparations containing different amounts of lauric acid exhibited different degree of inhibition against $M$. tuberculosis. The result of the study suggests the 
potential of VCOs agent against the growth of M. tuberculosis Similar effect on other organisms is a possibility and should also be explored. But more studies are needed to replicate the studies using different concentrations of VCOs, lauric acid and other fatty acids in VCO such as myristic acid, caproic or caprylic acids.

Key words: Lauric acid, virgin coconut oil, tuberculosis, food supplement

\section{BACKGROUND:}

Despite the availability of efficacious treatment, tuberculosis (TB) remains one of the major public health problems in the world. The World Health Organization (WHO) estimated over 8.8 million incident cases of TB in $2010(140 / 100,000)$, of which 3.9 million $(62 / 100,000)$ were smear-positives. An estimated 1.1 million people died from TB in 2010 among Human Immunodeficiency Virus (HIV)- negative cases of TB and additional 0.35 million deaths among HIV positive cases (1). The Philippines ranks ninth among the 22 high TB-burden countries in the world and $3^{\text {rd }}$ among the seven high-TB burden countries in the Western Pacific Region (1). Although tuberculosis is considered a curable disease, it ranks 6th among the top leading causes of mortality and morbidity in the Philippines (2).

Based on reports from the Department of Health (DOH), there has been significant progress in the implementation of the Directly Observed Short- course Therapy for TB (DOTS) from 1996 to late of 2003 in the country. However, Dr. Woojin Lew, medical officer at WHOPhilippines, said the Philippine government has "a long way to go" in terms of detecting, treating and curing highly infectious TB patients (2). He further expressed that highly infectious TB patients remains high in the Philippines $(2,3)$. Locally, 75 people are dying every day from the disease. $(2,3)$. The National Tuberculosis Control of the Philippines (NTP) has demonstrated two components of DOTS which are significantly related to improved clinical outcomes. These are government commitment to the program and continuous supply of anti-TB drugs to patients. In a country where drug supply and reliable distribution continue to be a problem and poor sanitation and nutrition increase the probability of re-infection, adjunctive management for the control of TB can greatly improve its control.

A clinical study by Dayrit showed that Virgin Coconut Oil (VCO) beneficially reduced viral load among six AIDs patients confined in a Referral Hospital for Infectious Diseases in Manila. Although none of the study subjects survived at the end of the study, this was the first study on VCO for HIV patients in the country (4). Enig similarly reported that lauric acid, a medium-chain fatty acid that comprises $50 \%$ of the fatty acids in coconut fat is converted by the body into "monolaurin" $(5,6)$. This derivative is believed to be responsible for the anti-viral properties of VCOs. As a precursor of monolaurin, lauric acid was shown to disrupt the lipid membranes of enveloped viruses. It is believed that monolaurin dissolves the lipids in the viral envelope causing their disintegration. It can also inactivate pathogenic bacteria, such as, Listeria monocytogenes, Helicobacter pylori and recently Staphylococcus aureus, protozoa, like Giardia lamblia, yeast, such as, Candida albicans and other fungi $(7,8,9)$. Other studies have shown that monolaurin may be useful in the prevention and treatment of severe bacterial infections, especially those that are difficult to treat or are already resistant to antibiotics $(8,9)$. 
Since Mycobacterium tuberculosis has a lipid structure in its cell wall, it was theorized that the principal unsaturated fatty acid of $\mathrm{VCO}$, lauric acid, is responsible for exerting the same antibacterial action exhibited by monolaurin on the walls of pathogenic bacteria.

The country is one of the biggest exporters of coconut oil. VCO export earnings which surged by 138.65 percent to $\$ 7.56$ million in January to March 2012 compared to the $\$ 3.17$ million recorded a year ago contribute significantly to the economy. Forbes reported that the volume of VCO exports also surged to 1,719.23 metric tons, 93.58 percent higher than the 888 MT recorded a year ago (10). Thus, it is important that any health benefits attributed to VCO must be scientifically validated in order to maintain viability of the sale and promotion of VCO. In this study, we investigated the in vitro potential of VCO coming from the fruit of a highly abundant and indigenous tree, the coconut. The purpose of the study was not to challenge the current efficacy of antibiotics for tuberculosis but to explore supplementary or adjunctive treatment for TB. The population expected to benefit most are patients residing in hard to reach communities which may have limited or inconsistent access to anti-TB drugs. Thus, it was important for us to firstly document any antibacterial activity of VCO against $M$. tuberculosis. To achieve this objective, we worked on two preparations of VCO produced by two extraction methods and containing different amounts of lauric acid. Using in vitro susceptibility tests, we measured their inhibitory effects against M. tuberculosis isolates compared to a preparation of $45 \%$ lauric acid alone and a negative control containing the M. tuberculosis isolates.

\section{MATERIALS AND METHOD:}

All microbiologic tests for the study were performed at the Medical Research Laboratory of the Section of Infectious Diseases of the UP-Philippine General Hospital. It is a major referral hospital in the Philippines. The technical working group was composed of a Pharmacologist, Pharmacist and Microbiologist who regularly met to address emerging problems during the actual conduct of the test. These meetings occurred quite frequently since the test product was not the regular standardized pharmaceutical preparation but rather a functional food.

The VCO samples were selected by the study trial monitor (Philippine Council for Health Research and Development, PCHRD) based on existing sale data of VCOs. In addition, we requested for VCO preparations produced by two extraction processes aside from having the highest sale in the market. The VCOs were submitted to the study site as coded specimens, namely VCO 013 and VCO 012.

A. Test samples: VCO 013 was described by the manufacturer as 100\% virgin and unrefined. Its product insert contains the following information about the preparation: "it is made from freshpicked coconuts and fresh-milled to attain a lauric acid content of 56\%". It was not subjected to heat and did not undergo any processing such as bleaching and deodorizing. It was produced naturally from the kernels of carefully selected Philippine coconuts". VCO 013 was prepared by the cold process-a no heat (ANH) process similar to the process of wet milling whereby oil is extracted from fresh coconut meat without drying first. Wet milling means, "coconut milk" is expressed first by pressing. "The oil is then further separated from the water. Methods which can be used to separate the oil from the water include boiling, fermentation, refrigeration, enzymes and mechanical centrifuge" (11). In the case of VCO 013, centrifuging was used. 
VCO 012 was made from "genuinely pure Virgin Coconut Oil extracted from the choicest, freshest, and organically-grown Philippine coconuts; produced through Fresh-Dry method at the lowest temperature possible without chemical intervention. This is also known as "dry milling". The coconut meat was quick dried, and the oil was then pressed out via mechanical means. This is the most common type of "Virgin" or "Extra Virgin" coconut oil sold in the market today. Except for the fruity odor of VCO 012, the color, consistency and general appearances of both preparations were indistinguishable. Claims have been made that the less heat used in producing VCO, the higher the content of antioxidants (12). The study group assumed that if there was a difference in antioxidants, difference in inhibiting the growth of the TB isolates might be observed.

Both VCOs were compared with $45 \%$ lauric acid. This concentration was based on the usual reported amount of lauric acid in local coconut oil ranging from 45-50\%. They were also compared with a negative control containing 7H9 Broth plus the TB organism [ H37RV]. Antimicrobial property of all the test samples and comparators was tested against H37RV strain of $M$. tuberculosis obtained from the Infectious Disease Section of UP Manila- Philippine General Hospital.

\section{B. Procedure:}

1. Preparation of the VCO test samples: The VCO test samples were prepared containing $0.5 \mathrm{ml}$ of the VCOs plus $5 \mathrm{ml}$ of $7 \mathrm{H} 9$ broth. Initial tests were performed on the emulsified preparations. However, the emulsifying agent, CDEA, which was considered inert also inhibited growth of TB isolates in-vitro. Thus, the final test samples were the original forms sold commercially.

2. Preparation of H37RV inoculum: A frozen stock of $1 \times 10^{8} \mathrm{CFU} / \mathrm{mL}$ M. tuberculosis H37RV was thawed and diluted with 7H9 with Albumin, Dextrose and Catalase (ADC) to give a $1 \times 10^{4} \mathrm{CFU} / \mathrm{mL}$ concentration. $\mathrm{ADC}$ is an enrichment supplement to enhance the cultivation of Mycobacteria.

3. Preparation of the Middlebrook 7H10 Agar Plate: The Middlebrook 7H10 agar was prepared according to the manufacturer's instruction. The agar after sterilization was cooled in a water bath at $50^{\circ} \mathrm{C}$ to $56^{\circ} \mathrm{C}$. Five milliliters of the agar was dispersed in duplicate plates and allowed to solidify. The quadrant plates were placed in plastic bags and stored at $4^{\circ} \mathrm{C}$ to $8^{0} \mathrm{C}$. The prepared plates were used within 28 days after preparation.

4. In -vitro susceptibility test proper: Exactly $0.1 \mathrm{~mL}$ of the prepared H37RV $\left(1 \times 10^{4}\right.$ $\mathrm{CFU} / \mathrm{mL}$ ) was added to each tubes containing the VCO samples, negative control and lauric acid. The mixtures were incubated at $37^{\circ} \mathrm{C}$ for five days, with shaking for 5-10 minutes every hour during daytime. After five days, each of the mixtures was diluted 10 fold up to $10^{-4}$. The quadrant plates with Middlebrook 7H10 agar was inoculated with 0.1 $\mathrm{mL}$ of each dilution. The control plate was likewise inoculated with the corresponding diluted H37RV. The same procedure was repeated using Lowenstein Jensen (L-J) slants. All quadrant plates and L-J slants were incubated at $37{ }^{\circ} \mathrm{C}$, and examined for growth every week for 3 to 8 weeks. 
At the end of the incubation period, colonies on the quadrant plates and L-J slants were counted and recorded. Colony counts in the test quadrants (test preparations + organism) were compared with the counts in the control quadrants (organism + broth). As we performed more runs of the test, we noted the importance of ensuring longer contact time between VCO and the test organism. This was accomplished by continuous shaking and vigorous agitation during incubation. Additionally, the mixtures of $\mathrm{H} 37 \mathrm{RV}$ and the test samples were allowed to react for 5 days. Intermittent shaking for 10-15 minutes during daytime was done to further enhance the contact between organisms and test compounds. At the end of the incubation period, the test preparations were diluted 10-fold to ensure that the colonies which will grow can be counted and do not overlap.

\section{RESULTS:}

Percentage amount of lauric acid in the test VCOs: The usual composition of coconut oil described by the literature is listed in Table 1 juxtaposed with the fatty acids present in the two VCOs in our study. Because of limited resources, the composition of the VCOs were obtained from tests done by the manufacturer and was not validated by the study group. Both VCOs contained amounts of lauric acid within the range acceptable for coconut oils. However, VCO 013 contained $8.3 \%$ more of the lauric acid than the comparator VCO 012. Medical literature places the lauric acid content of most Virgin Coconut Oils at around 45-53\% (13).

Table 1. Composition of fatty acids in the VCOs used in the study

\begin{tabular}{|c|c|c|c|}
\hline $\begin{array}{l}\text { Common Name and } \\
\text { Composition } \\
\text { Percentage (\%) }\end{array}$ & Coconut oil & VCO 013 & VCO 012 \\
\hline Caproic & ND- 0.7 & 0.2 & 7.8 \\
\hline Caprylic & $4.6-10.0$ & 5.0 & 6.7 \\
\hline C 10:0 & $5.0-8.0$ & 4.7 & 0.5 \\
\hline C 12:0 & $45.1-53.2$ & 55.8 & 47.5 \\
\hline Myristic & $16.8-21.0$ & 18.4 & \\
\hline Palmitic $\quad$ C $16: 0$ & $7.5-10.2$ & 8.0 & \\
\hline Palmitoleic C 16:1 & ND & & \\
\hline Stearic & $2.0-4.0$ & 2.2 & \\
\hline C $18: 1$ & $5.0-10.0$ & 5.5 & \\
\hline Linoleic & $1.0-2.5$ & 1.1 & \\
\hline Linolenic $\quad$ C 18:3 & ND-0.2 & & \\
\hline Nervonic $\quad$ C 24 & ND & & \\
\hline
\end{tabular}

$\mathrm{ND}=$ non detectable

Not surprisingly, we observed greater inhibition of the growth of TB isolates by VCO 013 which contained more lauric acid than VCO 012. Please see Table 2. The same ability of VCOs to inhibit TB growth was demonstrated in the second replicate. We observed a much lower inhibitory effect of VCO 013 for the same concentrations than VCO 012. This might be attributed to a random error and would have been avoided if there were more replicates. 
Presently, we cannot provide an explanation for the results but it is possible that it is not the concentration of lauric acid per se that causes the antibacterial action of VCO but the ratio of a high lauric acid to the other fatty acids in coconut oils. Consistent though was the inhibitory effect of lauric acids in all replicates confirming the assumption that it might be the principle responsible for the antibacterial effect of coconut oils $(8,9,14)$.

Table 2. Colony count of TB in Middlebrook agar at increasing dilution of the control, VCO samples and lauric acid based on two replicates

\begin{tabular}{|c|c|c|c|c|c|c|c|c|}
\hline & \multicolumn{4}{|c|}{ Replicate 1} & \multicolumn{4}{|c|}{ Replicate 2} \\
\hline Dilution & Control & $\begin{array}{l}V C O \\
56 \% \\
\end{array}$ & $\begin{array}{l}V C O \\
47 \% \\
\end{array}$ & $\begin{array}{l}\text { Lauric } \\
\text { acid } \\
(100 \%) \\
\end{array}$ & Control & $\begin{array}{l}V C O \\
56 \% \\
\end{array}$ & $\begin{array}{c}V C O \\
47 \% \\
\end{array}$ & $\begin{array}{l}\text { Lauric } \\
\text { acid } \\
(100 \%) \\
\end{array}$ \\
\hline Undiluted & 560 & 560 & 560 & 0 & 560 & 560 & 560 & 0 \\
\hline $10^{-} 1$ & 560 & 85 & 300 & 0 & 560 & 369 & 560 & 0 \\
\hline $10^{-2}$ & 312 & 0 & 169 & 0 & 312 & 120 & 111 & 0 \\
\hline $10^{-3} 3$ & 89 & 35 & 33 & 0 & 89 & 100 & 44 & 0 \\
\hline $10^{-} 4$ & 103 & 1 & 0 & 0 & 103 & 16 & 15 & 0 \\
\hline
\end{tabular}

In the following Figure, average colony counts of M. tuberculosis isolates were used.

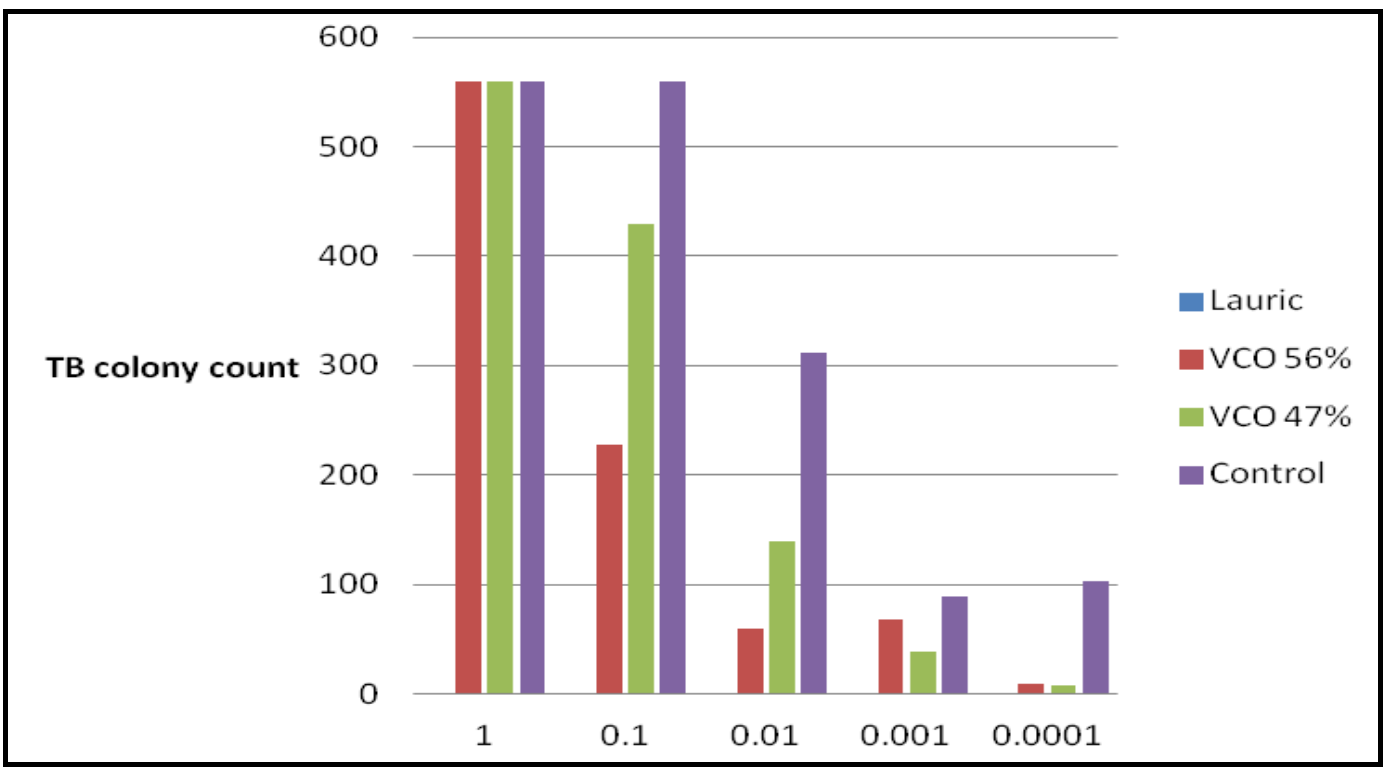

Figure 1. Average colony count of M.tuberculosis in Middlebrook agar at different dilution

Of the two VCO brands, VCO 013 showed early and significant decrease in the number of TB colonies starting from 1:10 dilution and progressively to almost complete inhibition of $M$. tuberculosis growth at 1:10000 dilution. Another set of susceptibility test was performed using L-J slants. Growth of M. tuberculosis in the L-J slant was more dispersed and easier to count. 
Table 3. Colony count of M. tuberculosis in LJ slants at increasing dilution of the control, VCO samples and Lauric acid

\begin{tabular}{|c|c|c|c|c|c|c|c|c|c|c|}
\hline \multirow[b]{2}{*}{ Dilution } & \multicolumn{5}{|c|}{$\begin{array}{c}\text { Replicate } 1 \\
\text { Colony count of TB }\end{array}$} & & \multicolumn{4}{|c|}{$\begin{array}{c}\text { Replicate 2 } \\
\text { Colony count of TB }\end{array}$} \\
\hline & I & $0^{-1}$ & $0^{-2}$ & $0^{-3}$ & $0^{-4}$ & & $0^{-1}$ & $0^{-2}$ & $0^{-3}$ & $0^{-4}$ \\
\hline $\begin{array}{l}\text { Control* } \\
(* \text { organism } \quad+7 \mathrm{H} 9 \\
\text { broth) }\end{array}$ & 300 & 0 & 4 & & 6 & 00 & 0 & 4 & & 6 \\
\hline VCO $56 \%$ & 84 & 02 & 0 & & & 50 & 2 & 5 & & \\
\hline$\overline{\mathrm{VCO}} 47 \%$ & 180 & 07 & 0 & & & 90 & 33 & & & \\
\hline Lauric acid & 0 & & & & & & & & & \\
\hline
\end{tabular}

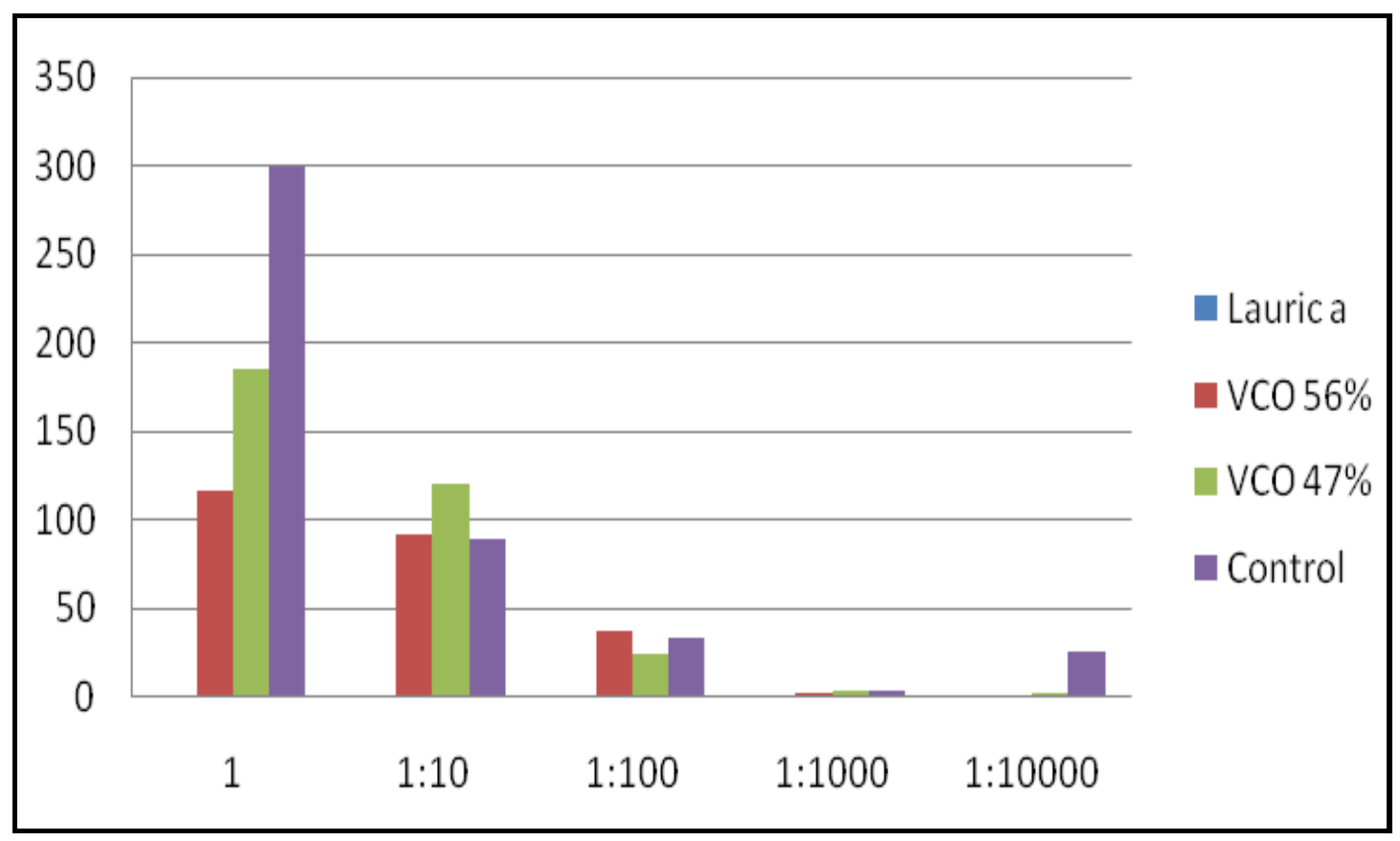

Figure 2. Average colony count of M. tuberculosis in LJ Slant in different dilutions

Using Middlebrook 7H10 agar, both VCO 012 and VCO 013 in 1:100 to 1:10000 dilution showed bacterial reduction or growth inhibition ranging from $46 \%$ to $100 \%$ respectively. The same trend was noted for bacterial growth in L-J slants, with degree of inhibition ranging from $25 \%$ to $96 \%$ shown in Table 3. The antibacterial effect of lauric acid was also confirmed in the L-J slant.

Statistical analysis: Being preliminary in nature, data merely underwent descriptive analysis. Data was run in MS Excel version 2007 and charts generated by the same. 


\section{DISCUSSION:}

Coconut oil is composed of triglycerides made of medium-chain and saturated fatty acids which is primarily saturated and medium chain fatty acids. Its $92 \%$ saturated fat content raises concern over possible cardiovascular risk. However, coconut oil has a $62 \%$ concentration of Medium Chain Fatty acids (MCFAs) which is a unique characteristic that is not present in other saturated fat" $(11,12)$. Unsaturated fat have a high concentration of long chain fatty acids unlike MCFAs which are easily absorbed by the body as it require less energy and fewer enzymes. Short and medium chain fatty acids are transported directly to the liver where they are immediately converted to energy (12). MCFA is suspected to prevent and heal a lot of sickness because of its antimicrobial properties. Aside from its ability to improve the immune system, MCFA also aids in the absorption and retention of magnesium, calcium and some amino acids (12).

Colonies of the TB organisms were noted to be confluent and impossible to count in all the undiluted test samples in both media. Thus, a count of 560 is often used to place a quantitative value to confluent growth. Colonies of $M$. tuberculosis could only be counted beginning at a dilution of 1:100. At this dilution, both brands of commercial VCOs showed inhibition of $M$. tuberculosis growth by as much as $46 \%$ to $100 \%$. Growth of M. tuberculosis decreased from baseline by $72 \%$ in the sample with the VCO 013 containing $56 \%$ of Lauric acid and by $40 \%$ for the VCO 012 containing $47 \%$ of lauric acid.

To confirm the viability of the test organisms, inocula taken from the mixtures of VCO and H37RV were inoculated into another medium, the Lowenstein Jensen (LJ) Medium. Grossly sufficient growth of the mycobacteria was shown in the LJ slant containing none of the VCOs and control materials. Results from the LJ slants also showed the inhibitory effects of both VCO 012 and VCO 013 on H37RV. However, literatures dealing on the L-J medium reported some limitations using the media on recovering isolates of $M$. tuberculosis (14). For example, a study conducted in India in 2005 compared the biphasic system (Middlebrook 7H11 agar slant + Middlebrook $9 \mathrm{H}$ broth) with Lowenstein - Jensen (LJ) medium. In smear positive cases, the biphasic system showed a recovery rate of $97.05 \%$ as against $79.41 \%$ on L-J on incubation for $21 \pm 4.44$ and $28 \pm 3.76$ days respectively. In smear negative and culture positive cases, the biphasic system and -LJ showed isolation rates of $91.66 \%$ and $66.6 \%$ after $36 \pm 3.44$ and $41 \pm 4.09$ days respectively. This suggests that the biphasic system showed lower contamination rate (1.33\%). It also concluded that the Biphasic medium is superior to L-J medium in isolating $M$. tuberculosis (15). But there was no difference between LJ slant and Middlebrook agar observed in our study.

Differences in inhibitory potential between the two commercial brands of VCO may be attributed to differences in the manner that they have been produced. This can be explained by studies showing different amount of oils based on different extraction methods. However, this was refuted by the study of Professor Dia (2005) of the University of the Philippines who studied three batches of VCO produced by three extraction methods. The values that were tested in all these VCOs were: melting point, gravity, saponification, iodine, free fatty acid (FFA), peroxide value, moisture content, fatty acid composition, tocopherols, and total phenolic content. Their results found that: "while the VCOs produced by the three methods and using different varieties had some differences in chemical and quality properties, these differences may not be large enough to significantly affect the overall quality of the VCO” (16). There is no industry standard 
definition for "Virgin Coconut Oil" as there is in the olive oil industry for "Virgin" and "Extra Virgin" olive oil.

CONCLUSION AND LIMITATIONS: The study using in-vitro method to test virgin coconut oil, an oil- based substance, against M. tuberculosisr showed the potential of VCOs against infection from the mycobacterial species (H37RV). The same action was likewise noted with $45 \%$ lauric acid. However, the study is limited by the insufficient number of replicates. The actual lauric acid content for both brands of the VCO was not validated by actual assay. Results of the agar and L-J slant showed consistent trend for inhibiting growth of TB isolates by both VCOs independent of the brand. Greater inhibition was observed with VCO 013 containing 56\% lauric acid than VCO 012 with $47 \%$ lauric acid during the initial run. This observation was reversed on the second run in favor of the latter. VCO with more lauric acid content showed earlier onset of action. Further studies are needed to determine the effective and lethal dose of VCO among humans. The possible anti-tuberculosis action of VCO in-vitro opens the door for clinical trials of other indigenous plant product against tuberculosis or as an adjunct to TB therapy. Prior to the discovery of streptomy cin, tuberculosis was partially eradicated by non pharmaceutical interventions such as improvement in sanitation and nutrition. It is not far fetched to expect VCO, which is rich in protein, to enhance the body's response to antibiotics. However, more studies are needed using varying and precise doses of lauric acid and VCOs in patients with tuberculosis. Randomized clinical trials adding VCO to the usual anti-TB drugs should be conducted and clinical responses of patients, such as early microbial cure, improvement of nutritional state and treatment success must be documented.

Competing interests: The authors declare that they have no competing interests.

Authors' contribution: Godofreda V. Dalmacion, MD, is the principal investigator of the study who conceptualized and formulated the research proposal and actively sought the funding to make the study possible. She also relentlessly worked to write the research article for publication. Being also an epidemiologist, she provided the analysis. Adelwisa R. Ortega, MD, is the microbiologist of the group whose critical review showed us the implications of the results of the study. Imelda G. Pena, DrPH is the Pharmacist of the group who was responsible for initially reconstituting the VCO preparations into more immiscible forms. She also was responsible for preparing all the test samples. Concepcion F. Ang, RMT is the Medical Technologist of the group who performed all the laboratory work.

Acknowledgement and Funding: We wish to thank the Department of Science and Technology-Philippine Council for Health Research who provided the funds to make this study possible. The authors are also grateful to the invaluable contributions of the following people to this study:

Dr. Jaime Montoya and Ms. Rose Taguiang of the Philippine Council for Health Researchand Development, Department of Science and Technology;Dr. Jaime Galvez-Tan, Former Executive Director of the National Institutes of Health, University of the Philippines Manila; Dr. Perla 
Santos-Ocampo, National Scientist and Former Chancellor, University of the PhilippinesManila, The Health Sciences Center.

\section{REFERENCES:}

1. World Health Organization (2005) TB control in Southeast Asia and Western Pacific Regions: a bi-regional report. Geneva, World Health Organization.

2. Ten leading causes of mortality by sex, Philippines, 2003. http://doh.gov.ph/data_stat/html/mortality.htm (accessed 08 March 2008).

3. World Health Organization (2011) Global Tuberculosis Control 2011. http://who.int/tb/publications/global_report/2011/gtbr_full.pdf

4. Ten leading causes of morbidity in 2003, Philippines. http://doh.gov.ph/data_stat/html/morbid.htm (Accessed 08 March 2008).

5. Dayrit, Conrado. Coconut oil in health and disease: Its monolaurin's potential as cure for HIV/AIDS. http://doh.gov.ph/sars/coconut_oil.htm (Accessed 11 March 2008).

6. Enig, Mary. Coconut: In Support of Good Health in the 21st Century. www.coconutoil.com/coconut_oil_21st_century.htm (accessed 01 Nov 2008).

7. Bergsson G, G.Arnfinnsson J, Steingrimsson O, Thirmar H: Killing of Gram-positive cocci by fatty acids and monoglycerides. APMIS 2001;109:670-678.

8. Kabara,J: Toxicological, bactericidal and fungicidal properties of fatty acids and some derivatives. Journal of American Oil Chemical Society 1984; 61:397-403.

9. Enig, Mary. The latest studies on coconut oil.www.westonaprice.org/knowyourfats/ coconut-oil-studies.html (accessed 01 Nov 2008).

10. Galvez J: Q1 VCO exports up by at least 200 percent. The Manila Times.net. www.manilatimes.net/index.php/business/top_business_news/25136-q1_ Vco_exports_up_ar_least_200_percent (Accessed 26 August 2012).

11. TB News and Update. Philippine Tuberculosis Society 2009. www.124.107.59.58/index.php?option_com_center\&view=article\&id=8\&Itemid=14 (Accessed 24 August 2012).

12. VCO: keywords: ANH:CProc.Curezone.com Educating instead of medicating. www.curezone.com/forums/an.asp?i=663425 (Accessed 24 August 2012).

13. Fatty acids composition of virgin coconut oil 2010. http://www.thevirgincoconutoil.com/articleitem.php?articleid=163 (Accessed 24 August 2012).

14. Ogbolu DO, Oni AA, Daini OA,Oloko AP. In -vitro antimicrobial properties of coconut oil in candida species in Ibada, Nigeria. JMed Food 2007 June 10(2):384-7

15. Ghatole M, Sable C, Kamale P, Kandle S, Jahagvidar V, Yemul V: Evaluation of Biphasic Culture System for Mycobacterial Isolates from the Sputum of Patients with Pulmonary Tuberculosis. Indian Journal of Medical Microbiology 2005; 23:111-113.

16. Dia, Vermont P (2005). Comparative Physiochemical Characteristics of Virgin Coconut Oil. The Philippine Agricultural Scientist Dec 2005; 88:4:462-475. 